\title{
Design and modeling of an autonomous multi-link snake robot, capable of 3D-motion
}

\author{
Rabel Rizkallah ${ }^{1}$, Najib Metni ${ }^{1, a}$, Jessica Mouawad ${ }^{1}$ and Ramy Saad ${ }^{1}$ \\ ${ }^{1}$ Notre Dame University - Louaize, Zouk Mosbeh, Lebanon
}

\begin{abstract}
The paper presents the design of an autonomous, wheeless, mechanical snake robot that was modeled and built at Notre Dame University - Louaize. The robot is also capable of 3D motion with an ability to climb in the z-direction. The snake is made of a series links, each containing one to three high torque DC motors and a gearing system. They are connected to each other through Aluminum hollow rods that can be rotated through a $180^{\circ}$ span. This allows the snake to move in various environments including unfriendly and cluttered ones. The front link has a proximity sensor used to map the environment. This mapping is sent to a microcontroller which controls and adapts the motion pattern of the snake. The snake can therefore choose to avoid obstacles, or climb over them if their height is within its range. The presented model is made of five links, but this number can be increased as their role is repetitive. The novel design is meant to overcome previous limitations by allowing 3D motion through electric actuators and low energy consumption.
\end{abstract}

\section{Introduction}

The development of snake-like robots has been a major trend for the past few years. Imitating nature in the design of robots greatly increase their ability to move on various terrains and surfaces. This comes handy in exploration missions where irregular surfaces of various types may be faced. Snake-like robots present many advantages because of their serpentine motion which allow them to move over most surface types and in clustered environments [1]. Wheeless snake robots have the important advantage of being much more solid and less prone to break downs on irregular surfaces, as compared with other wheeled robots. Snake robots can be used in places either too small for people to reach or too dangerous, or as a preliminary exploration before sending people like in space exploration missions. Hence, multiple industrial, scientific or military applications can be carried out by snake robots. For instance, dealing with the nuclear reactors of Fukushima is being carried with robots and snake robots in particular, as humans can't approach the highly radioactive facility yet $[2,3]$. Also, the ESA has developed a snake-like robot for a Mars exploration mission, when the first human mission to Mars is expected to happen in about ten to twenty years from now [4].

Snake robots should have a motion flexibility that would allow them to move along a serpanoid curve [1]. There are four major gaits when it comes to snake motion: serpentine, concertina, sidewinding and rectilinear $[1,5]$. Adopting these gaits is equivalent to emulate snakes' motion.
Several snake robots have been built. The world's first snake robot was developed by Hirose in 1972. The robot was large and heavy (weighing $350 \mathrm{~kg}$ ), was equipped with passive wheels and had a train-like appearance $[1,5]$. Several other snake robots with passive wheels have been proposed over the years. However, they are only able to move across relatively flat surfaces since passive wheels do not move very well in a cluttered environment. Completely wheeless snake robots usually consist of straight links interconnected by motorized joints. The Kulko snake robot is one example. Its outer surface is built by curved smooth surfaces to allow gliding, and is equipped by force sensors to detect the irregularities of the terrain so that the robot "senses" its environment [1]. While it's great in creating a serpentine motion and move in an obstacle-rich environment, it is relatively slow, and isn't meant to climb over these obstacles and move in the Z-direction.

The University of Michigan developed an OmniTread serpentine robot that has a pneumatic joint actuation system. Its body was covered on all surfaces by propulsion elements (tracks) which made the robot indifferent to rolling over $[6,7]$. This robot however relies on pneumatic joints and doesn't follow a serpentine curve.

Today's snake robots are still in their research phase and most of them are limited to 2D motion, or are too large, or have a very reduced operating life before needing to be recharged, etc... Also, motion in obstaclecluttered environments is still very difficult to achieve and modeling of such motion is the subject of many studies in labs and research centers around the world.

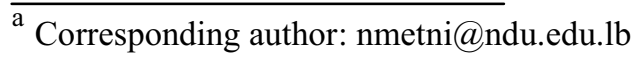


Our proposed solution adopts a novel design for the snake robot that will be presented in this study. This innovative new design allows 3D motion for an autonomous multi-link and wheeless snake robot. The multiple links of the robot are controlled electrically, as opposed to the popular pneumatic joints type. The study also tackles the 3D motion ability and ensures a fairly long life. Because of the elliptical shape of the links, some of them will present important free spaces which are used to insert the electric circuit and sensor on one part, and the batteries (large ones) on another part. The motion of the snake robot is a combination of serpentine and sidewinding motion [5] (Fig.1).

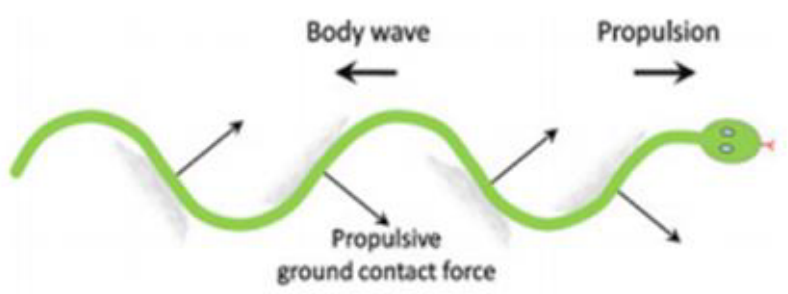

Lateral undulation.

Figure 1. Serpentine motion

The following sections of this paper will explain the general motion of the snake robot before describing its mechanical design and the function of each of the five links of the snake. Then, the mathematical model of the snake will be carried out, and a brief motion planning described. The paper ends on a conclusion and a description of the future works intended to further develop this new design.

\section{The snake robot design}

\subsection{Understanding the motion}

The described snake robot is made of five links. This number can be increased, as the role of these links follows a well-defined pattern. However the minimum number of links to achieve a reliable steady serpentine motion is five.

The actual motion of the snake robot is easily explained by looking at the sketch of Fig. 2 and 3. The links are numbered from 1 to 5 , where link number 1 designs the head of the snake, that is, the link where the vision sensor is placed.

It can be concluded that links 2 and 4 aren't needed to contribute to the rolling motion as links 1,3 and 5 are enough for this job. Instead, links 2 and 4 are used as joints, that is, to control the orientation of the links and therefore the direction of motion. In other words, links and joints are merged in this design, and there aren't any separate joint from the links.
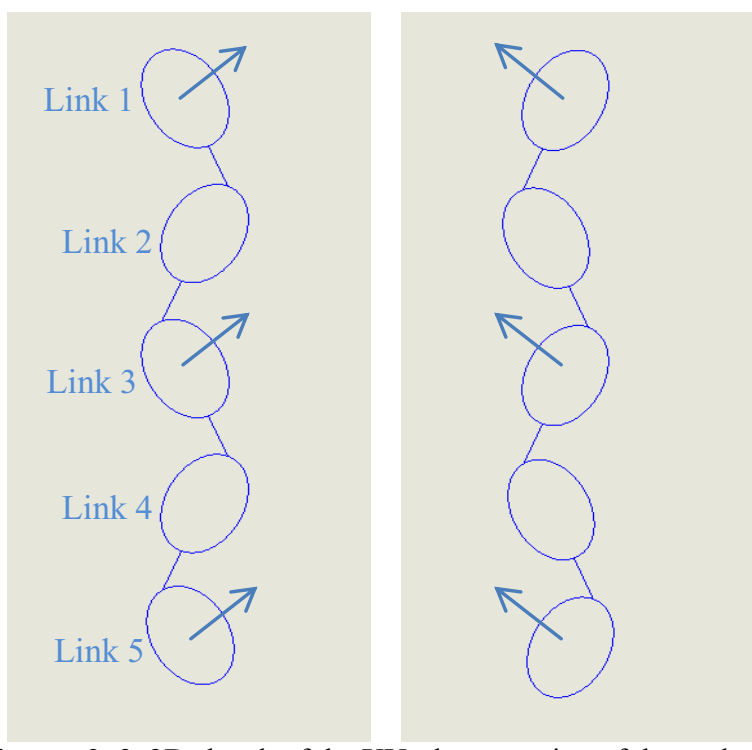

Figures 2, 3. 2D sketch of the XY-planar motion of the snake

When moving in the third dimension (the Z-direction), the rolling motion of links 3 and 5 isn't affected, and the snake can continue to move toward an obstacle even if both links 1 and 2 are raised in the air. Indeed, the joining system allows a complete separation between the XYplanar motion and the Z-motion.

\subsection{Model design}

The five links of the snake robots have an ellipsoid shape with openings at both ends. The links are connected together with Aluminum shafts.

Links 1, 3 and 5 rotate about themsleves generating the motion of the snake, while links 2 and 4 play the role of joints that control the rolling links. When the links are rotating, the connecting shafts don't rotate with them as they are attached to the links with bearings. It follows that links 2 and 4 also don't rotate. The rolling motion of links 1,3 and 5 is created by the use of an internal gear attached in a groove in their centres (Fig. 4). This gear is rotated by a mating gear driven by a motor that is fixed to the connecting shaft. The robot is able to detect obstacles with a proximity sensor placed on the tip of link 1 . Another proximity sensor can be placed on link 5, thus allowing the robot to move indifferently forward and backward.

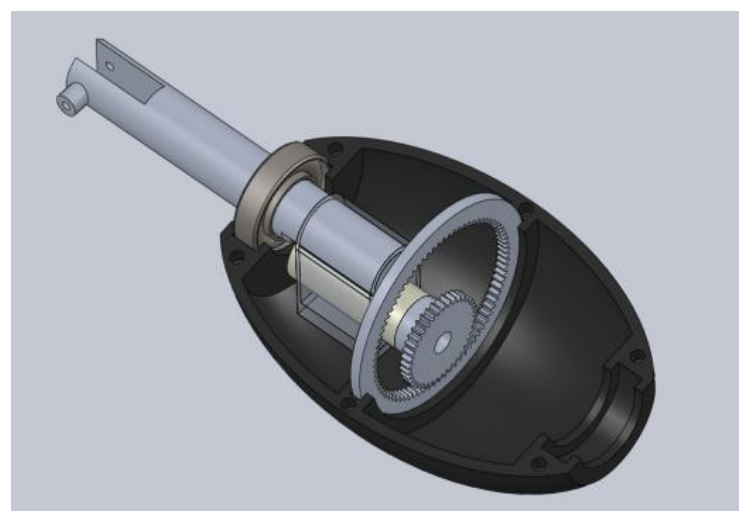

Figure 4. Interior view of links 1 and 5 
Links 2 and 4 contain the system needed to move the rolling links in the XY-direction (Fig. 5), and to move links 1 and 3 in the Z-direction.

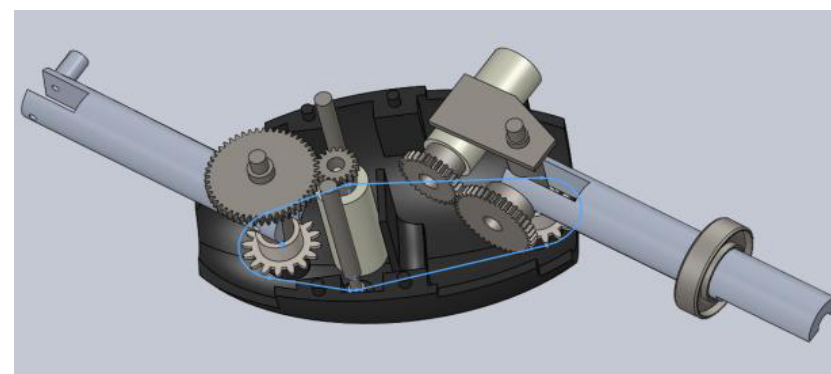

Figure 5. Interior view of links 2 and 4 containing the joint system

The XY-rotations of links 1, 2 and 3 are carried out by a chain and sprocket system with a ratio of $1: 1$. This ensures the angle between links 1 and 2 and the angle between links 2 and 3 are identical. The use of this sprocket and chain system instead of two independent motors followed space constraints, as using two motors would have resulted in the necessity of increasing the size of the links considerably. Similarly, the XY-rotations of links 3, 4 and 5 follows the samee logic. The XY-motors (the vertical motor in Fig. 5) are operated by the microcontroller and work together to avoid any mechanical failure of the chains and sprockets, in the case for instance that one motor would rotate in the opposite direction that the other.

Link 3 has a rolling motion similar to link 1 and is responsible for the Z-direction of link 2 (Fig. 6).

For this 5-links prototype, only links 1 and 2 can move in the Z-direction. Instead, the free space of link 5 is used to add the printed circuit, the microcontroller and the batteries. The assembled snake by combining all links is shown in Fig. 7.

A final note is that the connecting Aluminum shafts are hollow to allow for the wires to pass through the links till the controlling circuit in link 5 .

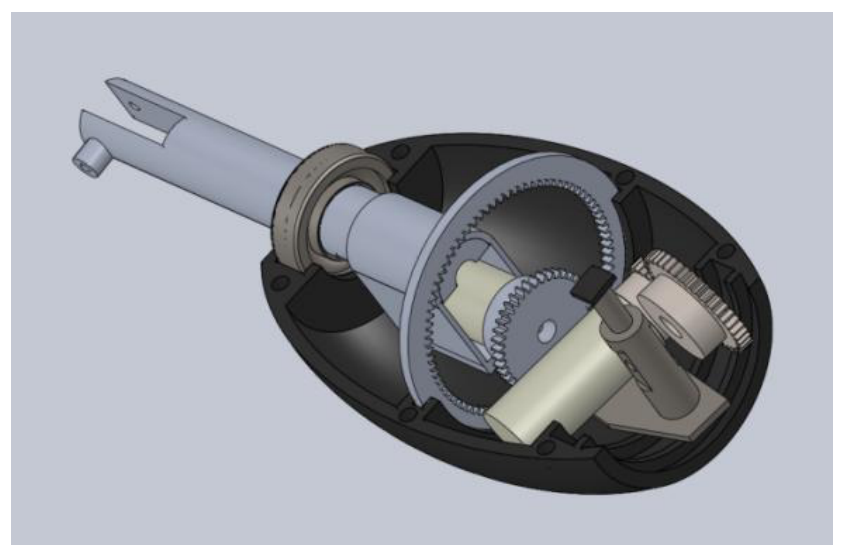

Figure 6. Interior view of link 3

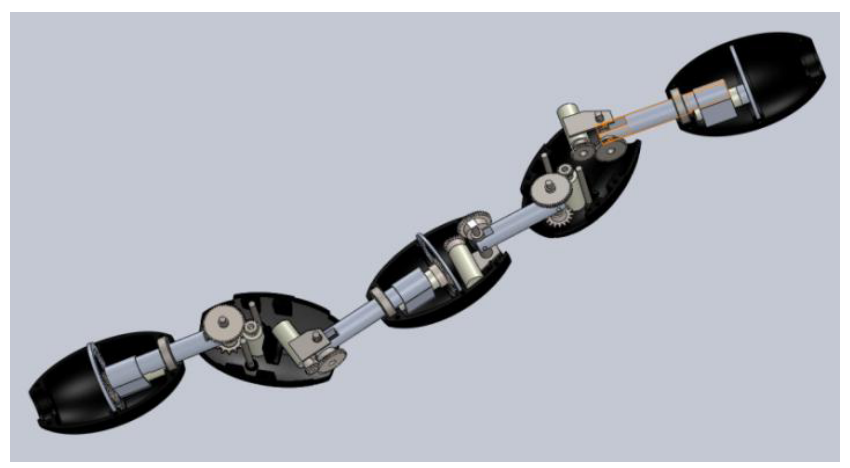

Figure 7. Interior view of the assembled snake robot

\section{Mathematical modeling}

Obtaining a fully defined set of equations of motion for the links that would relate them to the applied torques is desirable, as one would wish to know what torques the motors should deliver in order for the robot to reach a particular destination point. For this purpose, velocity propagation from link to link is used to obtain the Jacobian matrix, and a Lagrangian approach follows to expand equations of motion through the generalized degrees of freedom [8].

\subsection{Rotational matrices}

A first step is to define the rotational matrices of all links with respect to a fixed base frame, for instance, the ground. The convention of assigning frames to all links is that the $\mathrm{X}$-axis will point out of the link in the forward direction, the $\mathrm{Z}$-axis points upward away from and perpendicular to the ground and the $\mathrm{Y}$-axis completes the right hand rule [8] (Fig. 8).

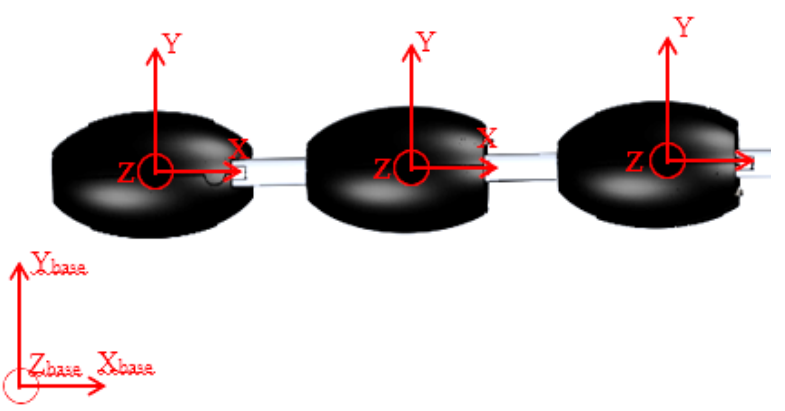

Figure 8. Frame assignment

Now that the frames are assigned, rotational matrices are defined for each link as seen in the base frame, using the Euler angles convention $[8,9]$. The angle $\theta$ defines the rotation around the $\mathrm{X}$-axis for each link, the angle $\alpha$ is the $\mathrm{XY}$-displacement and the angle $\gamma$ is the Z-displacement. As previously mentioned, link 1 is able to rotate about $X$, $\mathrm{Y}$ and $\mathrm{Z}$ axis. Link 2 rotates only in the $\mathrm{Z}$-direction (about $y$ axis). Link 3 rotates about itself and in the XYdirection ( $X$ and $Z$ axis). Link 4 rotates only in the $X Y$ direction (about the $Z$ axis), this because of the motion coming from link 3 and its own chain and sprocket system. Finally link 5 rotates about itself and about the Z- 
axis in the XY-plane. In other words, link 1 has $\theta_{1}, \alpha_{1}$ and $\gamma_{1}$ as corresponding degrees of freedom, link 2 has only $\gamma_{2}$, link 3 has the same $\alpha_{1}$ of link 1 with its own $\theta_{3}$, link 4 has $\alpha_{4}$ only and link 5 has the same $\alpha_{4}$ of link 1 with its own $\theta_{5}$. There are therefore seven degrees of freedom given by:

$$
\boldsymbol{q}=\left[\begin{array}{l}
\theta_{1} \\
\alpha_{1} \\
\gamma_{1} \\
\gamma_{2} \\
\theta_{3} \\
\alpha_{4} \\
\theta_{5}
\end{array}\right]
$$

The rotational matrices are of the form $[8,9]$ :

$$
{ }_{i}^{0} R=\left[\begin{array}{lll}
C_{1} & C_{2} & C_{3}
\end{array}\right]
$$

where the indices $i$ and 0 indicate that the matrix describes the rotation of the frame of link $i$ as seen in the base frame or frame $0[8]$, and where

$$
\begin{gathered}
\boldsymbol{C}_{\mathbf{1}}=\left[\begin{array}{c}
\cos \alpha_{i} \cos \gamma_{i} \\
\sin \alpha_{i} \cos \gamma_{i} \\
-\sin \gamma_{i}
\end{array}\right] \\
\boldsymbol{C}_{\mathbf{2}}=\left[\begin{array}{c}
\cos \alpha_{i} \sin \gamma_{i} \sin \theta_{i}-\sin \alpha_{i} \cos \theta_{i} \\
\sin \alpha_{i} \sin \gamma_{i} \sin \theta_{i}+\cos \alpha_{i} \cos \theta_{i} \\
\cos \gamma_{i} \sin \theta_{i}
\end{array}\right] \\
\boldsymbol{C}_{\mathbf{3}}=\left[\begin{array}{c}
\cos \alpha_{i} \sin \gamma_{i} \cos \theta_{i}+\sin \alpha_{i} \sin \theta_{i} \\
\sin \alpha_{i} \sin \gamma_{i} \cos \theta_{i}-\cos \alpha_{i} \sin \theta_{i} \\
\cos \gamma_{i} \cos \theta_{i}
\end{array}\right]
\end{gathered}
$$

Hence, ${ }_{1}^{0} \boldsymbol{R},{ }_{2}^{0} \boldsymbol{R},{ }_{3}^{0} \boldsymbol{R},{ }_{4}^{0} \boldsymbol{R}$ and ${ }_{5}^{0} \boldsymbol{R}$ are found, where the zero indicates that the rotational matrices are found with respect to the fixed base frame - the ground. Properties of rotational matrices [8,9] are used in Eq. (3) to find the rotation matrices from link to link in order to carry on with the velocity propagation.

$$
{ }_{i+1}^{i} R={ }_{0}^{i} R_{i+1}^{0} R={ }_{i}^{0} R^{T}{ }_{i+1}^{0} R
$$

\subsection{Velocity propagation}

Before proceeding with the velocity propagation as a method to derive the Jacobians [8] of all five of the links, we first define $l$ as the half length of the links, $d$ as the distance between the links and $a$ as the distance from the end of one link to the position of its joint's system's steel rod. These parameters are shown in Fig. 9.

With these defined, one can express the relative position of the center of each link with respect to the adjacent link. These are given in Eq. (4) till Eq. (7). Again, the indices mean that the matrix express the lower index's parameter in the frame of the upper index.

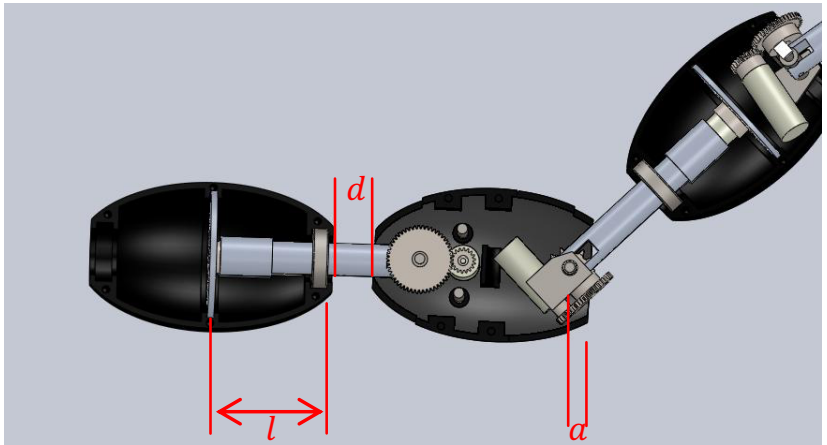

Figure 9. $l, d$ and $a$ parameters

$$
{ }_{2}^{1} \boldsymbol{P}=\left[\begin{array}{c}
-(l+d) \cos \gamma_{1}-l \cos \alpha_{1} \\
l \sin \alpha_{1} \cos \gamma_{1} \\
-(d+l) \sin \gamma_{1}
\end{array}\right]
$$

$$
\begin{gathered}
{ }_{3}^{2} \boldsymbol{P}=\left[\begin{array}{c}
-(l-a) \cos \gamma_{2}-(d+a) \cos \alpha_{1} \cos \gamma_{2}-l \cos \alpha_{1} \\
-(d+a) \sin \alpha_{1} \cos \gamma_{2}-l \sin \alpha_{1} \\
-(d+l) \sin \gamma_{2}
\end{array}\right] \\
{ }_{4}^{3} \boldsymbol{P}=\left[\begin{array}{c}
-l-(l+d) \cos \alpha_{4} \\
(l+d) \sin \alpha_{4} \\
0
\end{array}\right] \\
{ }_{5}^{4} \boldsymbol{P}=\left[\begin{array}{c}
-l-(l+d) \cos \alpha_{4} \\
(l+d) \sin \alpha_{4} \\
0
\end{array}\right]
\end{gathered}
$$

The physical quantities of the built 4-link prototype are given in the appendix.

The velocity propagation can now be carried on. Starting with the base frame, i.e., the ground, and taking in its own frame its linear velocity as ${ }_{0}^{0} v=0$ and its angular velocity as ${ }_{0}^{0} \omega=0$ since it is fixed, the velocity propagation for rotational joints based connections is found by applying Eq. (11) and Eq. (12) [8]:

$$
\begin{array}{r}
{ }_{i+1}^{i+1} v={ }_{i}^{i+1} R\left({ }_{i}^{i} v+{ }_{i}^{i} \omega \times{ }_{i+1}^{i} P\right) \\
{ }_{i+1}^{i+1} \omega={ }_{i}^{i+1} R{ }_{i}^{i} \omega+\left[\begin{array}{l}
\dot{\theta}_{i+1} \\
\dot{\gamma}_{i+1} \\
\dot{\alpha}_{i+1}
\end{array}\right]
\end{array}
$$

For instance, for links 1 and 2, the linear and angular velocity vectors are found to be:

$$
\begin{gathered}
{ }_{1}^{1} \omega=\left[\begin{array}{c}
\dot{\theta}_{1} \\
\dot{\gamma}_{1} \\
\dot{\alpha_{1}}
\end{array}\right] \\
{ }_{1}^{1} v=\left[\begin{array}{c}
\dot{\gamma_{1}}(l+d) \sin \left(\gamma_{1}\right) \\
r \dot{\theta}_{1}+(l+d) \dot{\alpha}_{1} \\
-\dot{\gamma}_{1}(l+d) \cos \left(\gamma_{1}\right)
\end{array}\right] \\
{ }_{2}^{2} \omega={ }_{1}^{2} R_{1}^{1} \omega+\left[\begin{array}{c}
0 \\
\dot{\gamma}_{2} \\
0
\end{array}\right] \\
{ }_{2}^{2} v={ }_{1}^{2} R\left({ }_{1}^{1} \omega \times{ }_{2}^{1} P+{ }_{1}^{1} v\right)+\left[\begin{array}{c}
\dot{\gamma}_{2}(l+d) \sin \left(\gamma_{2}\right) \\
0 \\
-\dot{\gamma_{2}}(l+d) \cos \left(\gamma_{2}\right)
\end{array}\right]
\end{gathered}
$$


The same procedure is carried on for links 3, 4 and 5 by applying Eq. (11) and Eq. (12).

These equations lead to finding the Jacobian in the frame of Link 5 with respect to the base frame. This is done by building the velocity matrix and isolating the rates of the degrees of freedom [8,9]. This gives a linear velocity Jacobian $\boldsymbol{J}_{\boldsymbol{v}_{\mathbf{5}}}$ coming from ${ }_{\mathbf{5}}^{\mathbf{v}} \boldsymbol{v}$ and an angular velocity Jacobian $\boldsymbol{J}_{\boldsymbol{\omega}_{\mathbf{5}}}$ coming from ${ }_{\mathbf{5}}^{\mathbf{5}} \boldsymbol{\omega}$.

$$
\mathbf{5} \boldsymbol{\omega}=\boldsymbol{J}_{\boldsymbol{\omega} \mathbf{5}}\left[\begin{array}{c}
\dot{\theta}_{1} \\
\dot{\alpha}_{1} \\
\dot{\gamma}_{1} \\
\dot{\gamma}_{2} \\
\dot{\theta}_{3} \\
\dot{\alpha}_{4} \\
\dot{\theta}_{5}
\end{array}\right], \quad{ }_{\mathbf{5}} \boldsymbol{v}=\boldsymbol{J}_{\boldsymbol{v}_{5}}\left[\begin{array}{c}
\dot{\theta}_{1} \\
\dot{\alpha}_{1} \\
\dot{\gamma}_{1} \\
\dot{\gamma}_{2} \\
\dot{\theta}_{3} \\
\dot{\alpha}_{4} \\
\dot{\theta}_{5}
\end{array}\right]
$$

The Jacobian in the frame of Link 5 with respect to the base is given by:

$$
J_{5}=\left[\begin{array}{l}
J_{v_{5}} \\
J_{\omega_{5}}
\end{array}\right]
$$

Obtaining the Jacobian $\boldsymbol{J}_{\mathbf{1}}$ in the frame of Link 1 is done by applying [8]:

Where

$$
J_{1}={ }_{5}^{1} R_{J} J_{5}
$$

$$
{ }_{5}^{\mathbf{1}} \boldsymbol{R}_{J}=\left[\begin{array}{cccccccc}
{\left[{ }_{1}^{0} \boldsymbol{R}^{T}{ }_{5} \boldsymbol{R}\right]} & & 0 & 0 & 0 & 0 & 0 \\
0 & 0 & 0 & 0 & 0 & 0 & 0 & 0 \\
0 & 0 & 0 & 0 & {\left[{ }_{1}^{0} \boldsymbol{R}^{T}{ }_{5}^{\mathbf{0}} \boldsymbol{R}\right]} \\
0 & 0 & 0 & 0 &
\end{array}\right]
$$

$\boldsymbol{J}_{\mathbf{2}}, \boldsymbol{J}_{\mathbf{3}}$ and $\boldsymbol{J}_{\mathbf{4}}$, the jacobians of links 2,3 and 4 respectively, are computed similarly.

\subsection{Lagrange method}

A Lagrangian approach is used to compute the equations of motion. This method that relies on the energy properties of the system has the advantage of requiring only kinetic and potential energies and the risk of committing errors is smaller than computing internal forces and summing inertial terms independently $[10,11]$.

The Lagrangian is defined as [10]:

$$
\mathcal{L}=T-V
$$

where $T$ is the kinetic energy of the system and $V$ is the potential energy.

For a multi-link robot, the kinetic energy $T$ can be computed in terms of the degrees of freedom by [8]:

$$
\boldsymbol{T}(q, \dot{q})=\frac{1}{2} \dot{q}^{T} \boldsymbol{M}(q) \dot{q}=\frac{1}{2} \sum_{i, j=1}^{n} M_{i j}(q) \dot{q}_{i} \dot{q}_{j}
$$

where $M(q)$ is the inertia matrix of the robot.

The potential energy $\mathrm{V}$ is computed as [11]:

$$
\boldsymbol{V}(q)=\sum_{i=1}^{n} m g h_{i}(q)
$$

In the case of the present snake robot, the potential energy is given by:

$$
V=g(d+l)\left[m_{1}\left(\sin \gamma_{1}+\sin \gamma_{2}\right)+m_{2} \sin \gamma_{2}\right]
$$

Applying the generalized Lagrange method to a multilink robot, the general formula for the equation of motion just discussed has the following form $[8,9]$ :

$$
\boldsymbol{M}(q) \ddot{q}+\boldsymbol{C}(q, \dot{q}) \dot{q}+\boldsymbol{N}(q, \dot{q})=\tau
$$

where

$$
\dot{\boldsymbol{q}}\left[\begin{array}{l}
\dot{\theta}_{1} \\
\dot{\alpha}_{1} \\
\dot{\gamma}_{1} \\
\dot{\gamma}_{2} \\
\dot{\theta}_{3} \\
\dot{\alpha}_{4} \\
\dot{\theta}_{5}
\end{array}\right], \quad \ddot{\boldsymbol{q}}=\left[\begin{array}{l}
\ddot{\theta}_{1} \\
\ddot{\alpha}_{1} \\
\ddot{\gamma}_{1} \\
\ddot{\gamma}_{2} \\
\ddot{\theta}_{3} \\
\ddot{\alpha}_{4} \\
\ddot{\theta}_{5}
\end{array}\right]
$$

- $M(q)$ is the robot inertia matrix defined as:

$$
\boldsymbol{M}(q)=\sum_{i=1}^{n} J_{i}^{T}(q) \mathcal{M}_{i} J_{i}(q)
$$

with $J$ being the Jacobian and $\mathcal{M}_{i}$ being the generalized inertia matrix for link $i$ defined by $[8,9]$ :

$$
\mathcal{M}=\left[\begin{array}{cc}
m I & 0 \\
0 & \mathcal{J}
\end{array}\right]
$$

where $m$ if the corresponding mass of link i, $I$ is a $3 \times 3$ identity matrix and $\mathcal{J}$ is the inertia tensor taken at the principle axis of the corresponding link according to the reference frames chosen.

- $\quad \boldsymbol{C}(q, \dot{q})$ is the Coriolis matrix defined as [8]:

$$
\boldsymbol{C}_{i j}(q, \dot{q})=\frac{1}{2} \sum_{k=1}^{n}\left(\frac{\partial M_{i j}}{\partial q_{k}}+\frac{\partial M_{i k}}{\partial q_{j}}-\frac{\partial M_{k j}}{\partial q_{i}}\right) \dot{q}_{k}
$$

- $\quad \boldsymbol{N}(q, \dot{q})$ represents the gravitational force and friction forces acting on the robot defined as [9]:

$$
-\boldsymbol{N}_{\boldsymbol{i}}(q, \dot{q})=-\frac{\partial V}{\partial q_{i}}-f_{i}
$$

Where the first term corresponds to the gravity effect on the robot, and $f_{i}$ is the friction force contribution given by the Coulomb model of friction. It is assumed that no friction contribution occurs with the snake moving in the Z-direction related coordinates, i.e., $\gamma_{1}$ and $\gamma_{2}$.

The friction vector is therefore given by the following:

$$
f=\mu \times g \times\left[\begin{array}{c}
\frac{1}{3}\left(m_{2}+m_{4}\right) \\
m_{1}+m_{2}+\frac{m_{3}}{2} \\
0 \\
0 \\
\frac{1}{3}\left(m_{2}+m_{4}\right) \\
\frac{m_{3}}{2}+m_{4}+m_{5} \\
\frac{1}{3}\left(m_{2}+m_{4}\right)
\end{array}\right]
$$

Now that all the necessary terms are defined, inserting them into Eq. (30) allows computing the equations of motion as a function of the applied torques and degrees of freedom. From here, a control law can be applied on the 
torques to control the motion of the robot. This is useful either for achieving a desired trajectory, or to satisfy a given constraint or avoid singularities for example. By controlling the torques, one can move the snake to a specific set of links' positions with respect to each other and at a specific point in the base frame.

\section{Motion planning}

Before starting its motion, the snake will map its environment using its proximity sensor at the tip of link 1 . For this, it will move link 1 in the XY-plane over its range and record the different entries the sensor will send based on the existence of obstacles, wall distances, etc.... Then link 1 will be raised in the Z-direction and moved again along its XY-range, to check for the height of potential obstacles. Based on the data collected, the microcontroller will choose the easier path for the snake to take. If it can stay in the XY-plane, then the snake will stick to $2 \mathrm{D}$ motion. If the obstacles encountered prevent the XY-motion, then the snake will climb over them by raising link 1, and link 2 if necessary and continuing to propel itself toward the obstacle by rolling links 3 and 5 . However, this only happens if the height of the obstacle is below 1.5 times the length of one link. Otherwise, the snake will try to turn around the obstacle and move away from it, as it cannot climb over it. Note that adding more links, and therefore allowing a greater number of them to move in the Z-direction would allow for the snake to climb over higher obstacles.

This mapping allows the snake robot to adapt to its environment and modify its trajectory accordingly. The mapping process occurs every time the snake travels $60 \%$ of the range of its proximity sensor. The distance traveled by the snake is computed by the microcontroller based on the rotation of the rolling links, as each of the angles of these links, their radii and the rotating speed of the controlling motors are known.

\section{Conclusion}

Snake robots nowadays became one of the most important research fields in robotics because of their ability to move over various types of surfaces. However, 3D motion and autonomy are still limitations in most of existing snake robots. The snake robot discussed in this paper is an original design that aims at allowing the $3 \mathrm{D}$ motion of the snake and achieving autonomy. The modeling of the robot is described, and using its seven degrees of freedom, a dynamic study of its motion was undergone using Jacobians and Lagrangian formulation. These calculations are a necessity to reach a control law that would allow controlling the torques applied at the joints to satisfy a certain desired trajectory or a certain constraint. The next step is to finish building the snake, as four links out of five have already been built and assembled, and implement the equation of motion on a computer robotic software to further develop the serpentine motion study and to optimize the design.references will be linked to their original source, only if possible. To enable this linking extra care should be taken when preparing reference lists.

\section{Appendix}

Table 1. Geometric properties of the 4-link built prototype

\begin{tabular}{|c|c|c|}
\hline$l$ & $d$ & $a$ \\
\hline $9.2 \mathrm{~cm}$ & $5 \mathrm{~cm}$ & $3.5 \mathrm{~cm}$ \\
\hline
\end{tabular}

Table 2. Inertia properties of all 4 links of the built prototype

\begin{tabular}{|c|c|c|c|c|}
\cline { 3 - 5 } \multicolumn{2}{c|}{} & \multicolumn{3}{c|}{ Principal Moments of Inertia $\left(\mathbf{g} / \mathbf{m m}^{\mathbf{2}}\right)$} \\
\hline Link & $\begin{array}{c}\text { Mass } \\
(\mathbf{k g})\end{array}$ & $\boldsymbol{I}_{\boldsymbol{x} \boldsymbol{x}}$ & $\boldsymbol{I}_{\boldsymbol{y} \boldsymbol{y}}$ & $\boldsymbol{I}_{\boldsymbol{z} z}$ \\
\hline 1 & 0.867 & 999120.09 & 3104354.37 & 3547134.25 \\
\hline 2 & 1.188 & 1179838.61 & 3220989.37 & 3272568.21 \\
\hline 3 & 1.160 & 1394570.99 & 4093972.93 & 4097743.85 \\
\hline 4 & 1.001 & 874121.6 & 1325211.58 & 1334195.75 \\
\hline
\end{tabular}

\section{References}

[1] Liljebäck, P. S. (2013). Snake Robots, Modeling, Mechatronics, and Control. Springer.

[2] Ackerman, E. (2015). Another Robot to Enter Fukushima Reactor, and We Wish It Were Modular. IEEE Spectrum.

[3] Ackerman, E. (2015). Unlucky Robot Gets Stranded Inside Fukushima Nuclear Reactor, Sends Back Critical Data. IEEE Spectrum.

[4] Ackerman, E. (2013). European Space Agency Wants to Send Robot Snakes to Mars. IEEE Spectrum.

[5] Y. K. Yansong Shan, "Design and Motion Planning of a Mechanical Snake," IEEE TRANSACTIONS ON SYSTEMS, MAN, AND CYBERNETICS, vol. 23, no. 4, pp. 1091-1100, 1993.

[6] Grzegorz Granosik, J. B. (2005). Integrated Joint Actuator for Serpentine Robots. Transactions on Mechatronics, 10(5), 473-481.

[7] Grzegorz Granosik, J. B. (June 23-25, 2005). The OmniTread serpentine robot with pneumatic joint actuation. Fifth International Workshop on Robot Motion and Control. Dymaczewo, Poland.

[8] J. Craig, Introduction to Robotics, Mechanics \& Control, Pearson- Prentice Hall, 2005.

[9] Murray, R. M. (1994). A Mathematical Introduction to Robotic Manipulation. CRC Press.

[10] Hand, L. N., \& Finch, J. D. (1998). Analytical Mechanics. Cambridge University Press.

[11] Teodorescu, P. P. (2009). Mechanical Systems, Classical Models. Bucharest: Editura Tehnica. 